\title{
Randomized clinical trial evaluating a digital gamified Acceptance and Commitment early intervention program for individuals at high-risk for eating disorders
}

\author{
Maria Karekla, Ph.D. ${ }^{1}$ Patrisia Nicolaou, Ph.D. ${ }^{1}$, \& Rhonda M. Merwin, Ph.D. ${ }^{2}$ \\ University of Cyprus, Department of Psychology; mkarekla@ucy.ac.cy; nikolaoupatrisia@hotmail.com \\ 2 Duke University Medical Center, Department of Psychiatry and Behavioral Sciences; rhonda.mer- \\ win@duke.edu \\ * Correspondence: Maria Karekla, Ph.D.; mkarekla@ucy.ac.cy; Tel.: 35722892100.
}

\begin{abstract}
Eating Disorders (ED) constitute a serious public health issue affecting predominantly women and appearing typically in adolescence or early adulthood. EDs are extremely difficult to treat as these disorders are ego-syntonic and many patients do not seek treatment. It is vital to focus on the development of successful early-intervention programs for individuals presenting at-risk and are on a trajectory towards developing EDs. This study is a randomized controlled trial evaluating an innovative digital gamified Acceptance and Commitment early-intervention program (AcceptME) for young females showing signs and symptoms of an ED and at high-risk for an ED. Participants $(\mathrm{N}=92$; Mage $=15.30$ years, $\mathrm{SD}=2.15)$ received either AcceptME $(\mathrm{N}=62)$ or a waitlist control $(\mathrm{N}=30)$. Analyses indicated that the AcceptME program effectively reduced weight and shape concerns, with large effects when compared to waitlist controls. Most participants scored below the atrisk cut-off (WCS score $<52$ ) in the AcceptME at end-of-intervention (57.1\%) compared to controls (7.1\%) with odds of falling into the at-risk group being 14.5 times higher for participants in the control group. At follow-up, $72 \%$ of completers reported scores below the at-risk cut-off in the AcceptME group. The intervention also resulted in a decrease in ED symptomatology and increased body image flexibility. Overall, results suggest that the AcceptME program holds promise for early-intervention of young women at-risk for developing an ED.
\end{abstract}

Keywords: Early-Intervention; High-risk for Eating Disorders; Acceptance and Commitment Therapy; Gamification; Digital Intervention; Vicarious learning.

\section{Introduction}

Prevention and early intervention programs for EDs to date have focussed on either reducing the pursuit of the thin ideal [1] or on disputing and replacing unrealistic thoughts with regard to food, body and weight [2]. These programs are based on the most influential cognitive behavioural theory of ED (CBT) and conceptualized as cognitive problems [3]. According to this approach, it is the individual's set of dysfunctional beliefs regarding the significance of weight and shape and an ED filter that biases perspective on the world that lead to pathology [3]. The CBT model of therapy focuses directly on disputing and replacing unrealistic thoughts with regard to food, body and weight [2]. An alternative approach is to consider the function of ED symptoms, and specifically the way in which they allow for avoidance and escape from unwanted internal experiences (e.g., negative affect generally, and specifically related to one's body) through symptom engagement and direct manipulation of the body [4-6]. Instead of attempting to alter cognitions, this approach would suggest teaching individuals to accept difficult thoughts and feelings, and engage in effective action (e.g., healthy eating) in the presence of emotions or other unwanted internal experiences [5]. 
Acceptance and Commitment Therapy (ACT) $[7,8]$ is a $3^{\text {rd }}$ wave CBT that targets ineffective internal control strategies and inflexibility found in a range of psychological problems. Rather than attempting to change internal experiences such as thoughts, sensations and feelings [8], ACT aims to change how individuals relate or respond to their thoughts/feelings. The aim is greater psychological flexibility, or to have unwanted internal experiences without unnecessary attempts to avoid or escape and without these experiences exerting undue influence over behaviour. ACT facilitates psychological flexibility via 6 core therapeutic processes: (1) experiential acceptance, (2) cognitive defusion, (3) present moment awareness, (4) self as context, (5) values clarification and living based on chosen values, and (6) committed purposeful action [9].

Accumulating evidence suggests that ACT holds potential for the treatment and prevention of EDs [4,10-12]. ACT has been shown to have efficacy for reducing ED symptoms relative to a waitlist control and treatment as usual (TAU) [13,14], and to have greater effects compared to cognitive therapy in one trial of ED symptoms secondary to anxiety or depression [15]. ACT has also been used with EDs across the age spectrum, including adolescents [16] and adults with ED symptoms [17] and for the spectrum of ED issues (e.g., restriction, binge eating; Lillis et al., 2011). A few studies have also found ACT to aid with maintenance of treatment gains and avoidance of hospitalization $[18,19]$.

Early evidence also supports increased acceptance (or more broadly, psychological flexibility) as a process of change in EDs. For example, studies have found low levels of acceptance are associated with ED symptom severity, and increased acceptance and body image flexibility (i.e., the ability to allow unwanted internal experiences, including negative thoughts/feelings about the body, without these experiences having undue influence over behavior) predicts symptom remission and moderates treatment response (e.g., BI-AAQ) $[20,21]$. There are also data suggesting that ED prevention programs that focus on body acceptance produce stronger effects relative to programs lacking this focus [1].

The vast majority of the evidence for ACT for ED studies have focused on individuals who were already exhibiting significant ED pathology. However, providing ACT skills early, when individuals have some ED signs and symptoms but before ED habits are formed, could alter illness trajectory. Yet, to our knowledge, there are not any ACTbased prevention or early-intervention programs that have been developed or tested for EDs.

EDs most commonly emerge between the ages of 16-20 years and previous studies have suggested that prevention and early intervention programs should target young women in this age range [1,22]. However, most young women with ED symptoms do not receive effective treatment [23]. There are several possible reasons for this, including that these individuals may choose low-threshold interventions instead of conventional health care provided for mental health problems [23]. Digital interventions have emerged in recent years and allow access to effective programs with lower costs, remove geographical constraints [24] and present the opportunity to cater to the interests and needs of youth with ED concerns. Such concerns include the desire for privacy or anonymity (due to high levels of guilt and shame) and access at all times of day and night [25]. Youth are also especially attracted to computer, console, and cell phone games, and there is a growing recognition that the use of digital gamification programs may be the preferred delivery method to reach today's youths [26]. Gamification is the application of game design elements into processes and services in order to engage and motivate [27]. Although the influence of gamification strategies to attract users, increase motivation and enhance learning is great, utilization of gamification components for behaviour change is still in its infancy. 
We developed an innovative digital, gamified ACT-based early-intervention program for high-risk for EDs adolescent girls (AcceptME; see XXX for a more extensive discussion of the treatment rationale- masked for blind review). This study tests this program in a two-arm randomized controlled trial. The AcceptME program presents with several innovations: a) it was based on ACT theory and practices; b) it used gamification principles to create a program appealing to youth and enhance learning through operant principles; c) it used a storyline in which the user helps a digital character overcome their personal difficulties related to ED-related problems (e.g., body weight and shape concerns), providing opportunities for vicarious learning; and d) it was a digitized earlyintervention program for individuals exhibiting symptoms of ED who have not yet crossed the diagnostic threshold. There are few programs that are developed explicitly for digital media and early intervention [28]. We hypothesized that participants receiving the AcceptME program, relative to a waitlist control, would evidence: a) lower weight concerns; b) fewer ED symptoms and behaviours; c) improved quality of life; d) decreased body dissatisfaction; and e) greater body image flexibility.

\section{Materials and Methods}

\subsection{Participants}

A total of 1050 young girls, 750 high-school students from twenty-five public high schools in Cyprus and 306 university students, aged 13 to 25 years $(M=16.8, S D=2.8)$ participated in the study. The majority of the sample were Greek-Cypriot (91\%), while 4\% were Turkish Cypriot, and 0.5\% were Maronite. Inclusion criteria were: females aged 1325 years, voluntary participation and parental consent for ages younger than 18 years, good working knowledge of the Greek language and score of 52 or greater on the Weight Concern Scale at screening [29], indicating early signs of an ED and high-risk for developing full threshold ED. Exclusion criteria were: possible current ED diagnosis as assessed by the Eating Disorder Diagnostic Scale (EDDS) [30].

\subsection{Procedure}

This is a two-arm clinical trial that registered at Clinicaltrial.gov (number

masked for review). Screening questionnaires were administered, either manually or via online computerized administration (i.e., SurveyMonkey, www.survey-

monkey.com/mp/audience) during class time and supervised by project researchers ( $\mathrm{N}=1050)$. Individuals identified as having a possible ED diagnosis (based on the EDDS) were referred to the Centre of Prevention and Treatment of Eating Disorders- Children and Adolescents Mental Health Services (only governmental clinic in the country offering these services). Individuals meeting criteria for participation (and their parents if $<18$ years of age; $\mathrm{N}=292$ ) were contacted via telephone and invited to participate in the trial. Of those invited, 92 agreed to participate and were randomized (2:1 randomization $A c$ ceptME vs. waitlist control). Of those, 62 were randomized to the AcceptME condition and were sent a text message with the webpage link, username, and password. Of those, 58 entered the program and completed the baseline assessment. Thirty were randomized into the control condition and were also asked to complete baseline measures. See Figure 1 for the Consort diagram of participant flow.

In addition to the baseline assessment, AcceptME participants completed assessments after each session, at end of intervention (EOI; i.e., immediately after completing Session 6) and at 1-month post-intervention follow-up. For every participant who completed the sixth session of AcceptME, one participant from the control group received a text message inviting her to complete the EOI assessment and provided with the option to then enroll to receive the intervention. 


\section{AcceptME program description}

AcceptME consisted of 6 sessions and each had to be completed within the same day it was began. Sessions were consecutive and participants had to complete them in order (see XXX (masked for blind review) for a video run-through of sessions. Three days after completion of a session, participants received a text message inviting them to continue to the next session.

The AcceptME program portrayed the story of a young girl contemplating whether to enter a reality television fashion contest. Participants followed the main character through making the decision to enter the contest and faced situations eliciting common challenging situations, thoughts and emotions, generally and especially related to her body image. A third person perspective was used, where the participant viewed the events unfolding from the perspective of an observer. Participants were encouraged to assist the leading character progressing through the game by completing exercises teaching and applying ACT skills (e.g., acceptance, defusion, present moment awareness, values consistent living) to progress in the contest and cope with distressing thoughts and feelings. Each session lasted approximately 30 minutes and included ACT experiential exercises and metaphors to teach core skills. The aim was for participants to develop new skills to be applied to their own life via helping the character achieve her goals. For a detailed description of the program content and development see XXX (masked for blind review).

\subsection{Measures}

Measures were completed by both the AcceptME and waitlist control group at baseline and EOI when the waitlist control group was offered access to the AcceptME program at that time. Participants in the AcceptME group completed the measures again at a 1month post-intervention follow-up point.

A demographic questionnaire assessed participants' age, nationality/ethnicity, school, and year in school/university.

The Weight Concerns Scale (WCS) [29] consists of 5 questions assessing fear of weight gain, worry about weight and body shape, importance of weight, diet history, and perceived fatness. Scores $>52$ are found to identify individuals at risk for later development (within the next 4 years) of an ED [29]. Killen and colleagues [29] reported satisfactory internal consistency (Cronbach's alpha $>.70$ ) and in this sample $a=.78$.

The Eating Disorder Diagnostic Scale (EDDS) [30] is a 22-item self-report scale of ED symptoms. It has shown high agreement with diagnoses made with the Eating Disorder Examination (EDE) [31] and is used to identify individuals meeting ED diagnosis criteria with high validity and reliability [32,33]. In this study, it was used to identify individuals who possibly meet criteria for ED for exclusion purposes. Based on the scoring syntax of Stice et al [30], scores indicating a possible ED diagnosis were excluded and referred for subsequent evaluation.

The Eating Disorder Examination Questionnaire (EDE-Q) [34] is a self-report version of the EDE interview, assessing frequency of key behavioural features and symptoms of eating disorders (i.e., number of times and days a behaviour has occurred in a 28-day period). Four subscales (Restraint, Weight Concern, Shape Concern and Eating Concerns) and a global score are derived. Score range is from 0-6 and higher global and scale scores suggest greater severity of ED pathology and more symptoms. Numerous studies support its psychometric validity and reliability [32-35]. Its Greek-version of EDE-Q presents adequate internal consistency similar to previous studies, with Cronbach's alphas for: 
Global=.78, Restraint=.74, Eating Concern=.91, Shape Concern=.91, and Weight Concern=.91 (Giovazolias, Tsaousis \& Vallianatou, 2012).

The Youth Quality of Life Instrument-Short Form (YQOL-SF) [36] is a self-report measure assessing the generic quality of life in youth aged 11-18 years. It includes 15 questions assessing domains of sense of self, social relationships, environment and general quality of life. The response scale ranges from 0 (not at all) to 10 (completely). The scores are summed and then transformed to a 0 to 100 scale, with higher scores representing higher quality of life. It has good internal consistency $(\alpha=.80)$ for all four domains and for the total score [36]. In this study, the Greek YQOL-SF presented with good internal consistency $(a=.75)$.

The Body Shape Questionnaire-8C (BSQ-8C) [37] is a self-report 8 question measure assessing body dissatisfaction and feelings of being fat. It presented with excellent treatment sensitivity to change in the course of therapy [38]. Questions refer to the participant's body dissatisfaction over the past four weeks, rated on a 6-point scale from "never" to "always". Higher scores indicate more body dissatisfaction. In the present study, the BSQ-8C Greek was found to have good internal consistency $(a=.87)$, similar to previous reports $(\alpha=0.93)$ [39].

The Body Image Acceptance and Action Questionnaire (BI-AAQ) [40] assesses psychological flexibility with body-image related thoughts and feelings, and specifically the ability to behave flexibly and effectively in the presence of thoughts and feelings about the body, without unnecessary attempts to avoid or escape these experiences. It is a unifactorial measures and it demonstrated excellent internally consistent $(\alpha=.93)$, good concurrent validity and good criterion-related validity [40]. The Greek-version also demonstrated high internal consistency across two samples $(a=.95)$ and was found to be a significant predictor of eating disorder behaviors after controlling for BMI and weight concern scores [41].

The Body Image Avoidance Questionnaire (BIAQ) [42] assesses behavioral tendencies of avoiding situations that trigger concerns about physical appearance. It also assesses other behaviors such as frequent weighting and inspection in the mirror. It was found to have good internal consistency (Cronbach's $\alpha=0.89)$ and test-retest reliability $(r=0.87)$ [42]. Similarly, the Greek-version in this study presented with good internal consistency $(a=.87)$.

\subsubsection{Statistical Analyses}

Firstly, we assessed equivalence of the intervention and waitlist control groups at baseline on demographics and outcome variables using a two-tailed independent sample ttests for age, and multiple one-way between-group analyses for treatment outcomes. Individuals from the AcceptME group who completed all six sessions and the end-oftreatment assessment were considered completers, whereas participants who completed at least one AcceptME session but did not complete it in its entirety were considered noncompleters. There was no missing data as the digital program required participants to complete each item before proceeding. To examine study hypotheses, a series of oneway Repeated Measures ANOVAs were conducted. Intent-to-treat analyses (ITT), with the last observation carried forward (i.e., baseline) method on participants' scores was also conducted for all significant findings. Finally, chi square and risk analysis were conducted to examine the odds of falling into the at-risk group (WCS $>52$ ) at end-of-intervention (EOI). All statistical analyses were conducted using SPSS (IBM SPSS v.27).

\section{Results}


The mean age of participants $(\mathrm{N}=89)$ enrolled in the trial was 15.30 years old $(\mathrm{SD}=2.15$; range: 13-22years old). Sixty-three percent of those randomized completed post-assessment (AcceptME=30, waitlist control=28) and were included in the primary outcome analyses. Twenty-five of the participants (83\% of the AcceptME group) competed the 1month follow-up.

\subsection{Group equivalence prior to intervention}

There was no statistically significant difference in age between the 2 groups (AcceptME: $\mathrm{M}=15.27$, $\mathrm{SD}=2.25$; waitlist control: $\mathrm{M}=15.09, \mathrm{SD}=1.89$ ). Multiple one-way between-group analyses indicated no significant differences $(\mathrm{p}>.05)$ between the AcceptME and waitlist control groups on baseline variables (i.e., weight concern, ED symptomatology, quality of life, body dissatisfaction, body image flexibility and avoidance).

\subsection{Comparison of outcome and process variable at baseline between program completers and non-completers}

Multiple one-way between-group analyses indicated no statistically significant differences ( $p>$.05) between the completers and non-completers for baseline outcome variables (i.e., weight concern, ED symptomatology, quality of life, body dissatisfaction, and body image flexibility and avoidance).

\subsection{Repeated Measures Analysis of Variance of Group by Time on Outcomes}

Means, standard deviations, pre-and post-data of all outcomes for both groups are summarized in Table 1. A series of one-way Repeated Measures ANOVAs were conducted to examine the effects of time (2: baseline vs. end-of-intervention (EOI)) and group (2: AcceptME vs. waitlist control) on outcomes.

There was a statistically significant interaction between time and group on participant's WCS scores, $\mathrm{F}(1,54)=56.67, \mathrm{p}<.001, \mathrm{n}^{2}=.52$. The main effect for time was not significant: $\mathrm{F}(1,54)=2.97, \mathrm{p}>.05, \mathrm{n}^{2}=.05$. There was however, a significant main effect for group: $\mathrm{F}(1,54)=12.16, \mathrm{p}<.001, \mathrm{n}^{2}=.95$, with the AcceptME group presenting with lower weight concerns $(\mathrm{M}=49.95, \mathrm{SD}=25.90)$ than the waitlist control group $(\mathrm{M}=76.54, \mathrm{SD}=14.85)$. Single degree of freedom interaction contrasts showed that differences driving the interaction were those at EOI, $\mathrm{F}(1,54)=24.40, \mathrm{p}<.001, \eta^{2}=.36$ (see Figure $2 \mathrm{a}$ ). The ITT results were similar showing interaction effect, $\mathrm{F}(1,85)=42.70, \mathrm{p}<.01, \mathrm{n}^{2}=.33$, main effect for time, $\mathrm{F}(1,85)=4.21, \mathrm{p}<.05, \mathrm{\eta}^{2}=.04$, and main effect for group, $\mathrm{F}(1,85)=15.76, \mathrm{p}<.01, \mathrm{\eta}^{2}=.16$. Single degree of freedom interaction contrasts showed significant differences between time, with the AcceptME group presenting significant decreases from baseline ( $M=57.08$; $\mathrm{SE}=2.26)$ to $\mathrm{EOI}(\mathrm{M}=49.77 ; \mathrm{SE}=2.77)$ and the control group presenting significant increases (baseline: $\mathrm{M}=62.00, \mathrm{SE}=3.12$; $\mathrm{EOI}: \mathrm{M}=76.00, \mathrm{SE}=3.74$ ).

There was a statistically significant interaction between time and group on participants' EDE-Q global scores, $\mathrm{F}(1,55)=6.80, \mathrm{p}=.01, \mathrm{\eta}^{2}=.11$ (see Figure 3a). Single degree of freedom interaction contrasts showed that differences driving the interaction were those from baseline to EOI for the AcceptME condition only, $\mathrm{F}(1,55)=9.99, \mathrm{p}<.01, \mathrm{n}^{2}=.15$ (waitlist control group changes from baseline to EOI, $\mathrm{p}>$.05). Similarly, there was a statistically significant interaction between time and group on participants' EDE-shape concern subscale scores, $\mathrm{F}(1,54)=8.49$, $\mathrm{p}<.01, \mathrm{\eta}^{2}=.14$ (see Figure $3 \mathrm{~b}$ ). Changes in the AcceptME condition from baseline to EOI and not in the waitlist control, were those that drove the interaction effect based on single degree of freedom interaction contrasts, $\mathrm{F}(1,54)=13.00, \mathrm{p}<.01, \mathrm{\eta}^{2}=.19$ (control group changes from baseline to EOI, $\mathrm{p}>.05$ ). The interaction between time and group on participants' EDE-Q weight concern subscale scores was marginally significant, $\mathrm{F}(1,54)=3.81, \mathrm{p}=.05, \mathrm{\eta}^{2}=.07$ (see Figure $3 \mathrm{c}$ ). Single degree of freedom contrasts 
showed that it was changes from baseline to EOI in the AcceptME group that drove this interaction effect, $\mathrm{F}(1,54)=5.60, \mathrm{p}=.02, \mathrm{\eta}^{2}=.10$. No statistically significant interactions were found for EDE-Q restraint subscale scores, $\mathrm{F}(1,54)=1.85, \mathrm{p}>.5, \mathrm{n}^{2}=.03$ and EDE-Q eating concern subscale scores, $\mathrm{F}(1,55)=1.08, \mathrm{p}>.5, \mathrm{\eta}^{2}=.02$. ITT analysis corroborated the findings, where for example for the EDE-Q global score, there was a significant interaction, $\mathrm{F}(1,86)=4.30, \mathrm{p}<.05, \mathrm{\eta}^{2}=.05$, and single degree of freedom interaction contrasts showing a significant decrease in ED symptoms in the AcceptME group from baseline $(M=2.20$; $\mathrm{SE}=.11)$ to $\mathrm{EOI}(\mathrm{M}=1.91 ; \mathrm{SE}=.11)$, whereas no differences were found for the control group (pre: $M=1.99, \mathrm{SE}=.16$; post: $\mathrm{M}=2.08, \mathrm{SE}=.15$ ).

No statistically significant interactions were found for body image flexibility, $\mathrm{F}(1,54)=1.86, \mathrm{p}>.5, \mathrm{\eta}^{2}=.03$. However, there was a significant time main effect $\mathrm{F}(1,54)=6.24$, $\mathrm{p}<.05, \eta^{2}=.10$ and single degree of freedom interaction contrasts showing that for the AcceptME group there was a significant increase in body image flexibility at EOI compared to baseline $(\mathrm{p}<.01)$, and this was not noted for the waitlist control group ( $\mathrm{p}>$.05). ITT analysis corroborated these findings.

There were no significant interactions for Quality of life $\left(\mathrm{F}(1,49)=.46, \mathrm{p}>.05, \mathrm{\eta}^{2}=.01\right)$, Body dissatisfaction $\left(\mathrm{F}(1,49)=3.78, \mathrm{p}>.5, \mathrm{\eta}^{2}=.07\right)$, and Body image avoidance, $(\mathrm{F}(1,49)=.22, \mathrm{p}>.05$, $\left.\eta^{2}=.00\right)$.

\subsection{Analysis across time (baseline, EOI, 1-month follow-up) for AcceptME group only}

Means, standard deviations of baseline, EOI and one-month follow-up assessments for outcomes are summarized in Table 2. A series of one-way Repeated Measures ANOVAs were conducted to examine the effects of time of the AcceptME group on outcomes.

The main effect of time was statistically significant on participants' WCS scores, $\mathrm{F}(2,22)=22.50, \mathrm{p}<.001, \mathrm{n}^{2}=.67$ (see Figure $2 \mathrm{~b}$ ). Pairwise comparisons indicated a significant difference on WCS mean scores between baseline and EOI $(\mathrm{p}<.01)$, as well as between baseline and 1-month follow-up $(\mathrm{p}<.001)$, but not between EOI and 1-month follow-up $(\mathrm{p}>.05)$.

The main effect of time was statistically significant on participants' EDE-Q global scores (see Figure $3 \mathrm{~d}$ ), $\mathrm{F}_{(2,23)}=14.93, \mathrm{p}<.001, \mathrm{\eta}^{2}=.57$. Pairwise comparisons indicated a significant difference on the EDE-Q global scores between baseline and EOI $(\mathrm{p}<.05)$ and 1-month follow-up $(\mathrm{p}<.001)$, and EOI and 1-month follow-up $(\mathrm{p}<.01)$. Regarding subscales, there was a statistically significant time-effect for the EDE-Q eating concerns, $F_{(2,23)}=12.14$, $\mathrm{p}<.001, \mathrm{\eta}^{2}=.51$. Pairwise comparisons indicated a significant difference between baseline and 1-month follow-up ( $\mathrm{p}<.001)$, and between EOI and 1-month follow-up ( $\mathrm{p}<.001)$, but not between baseline and EOI ( $\mathrm{p}>.05)$. The time effect for the EDE-Q shape subscale scores was also significant, $\mathrm{F}_{(2,23)}=9.65, \mathrm{p}<.001, \mathrm{\eta}^{2}=.46$, with the pairwise comparisons presenting differences between baseline and EOI $(\mathrm{p}<.05)$ and 1-month follow-up $(\mathrm{p}<.001)$, but there was no difference between EOI and 1-month follow-up ( $p>.05)$. Moreover, the main effect of time for EDE-Q weight concern subscale was statistically significant, $\mathrm{F}(2,23)=10.20, \mathrm{p}<.001, \mathrm{\eta}^{2}=.47$. Pairwise comparisons indicated significant differences between baseline and 1-month follow-up ( $\mathrm{p}<.001)$, and EOI and 1-month follow-up ( $<<.05)$, but not between baseline and EOI ( $\mathrm{p}>.05)$. The main effect for time was not statistically significant for the EDE-Q restraint scale.

The repeated measures ANOVA for body dissatisfaction (BSQ-8C scores) across time for the AcceptME group was statistically significant, $\mathrm{F}_{(2,23)}=13.38, \mathrm{p}<.001, \mathrm{\eta}^{2}=.54$. Pairwise comparisons indicated a significant difference between baseline and EOI scores $(\mathrm{p}<.01)$, baseline and 1-month follow-up $(\mathrm{p}<.001)$ and EOI and 1-month follow-up $(\mathrm{p}<.05)$. 
Similarly, the main effect of time was statistically significant on participant's BI-AAQ scores, $\mathrm{F}_{(2,23)}=10.40, \mathrm{p}<.001, \mathrm{n}^{2}=.48$. Pairwise comparisons indicated a significant difference between baseline and EOI scores $(\mathrm{p}<.05)$, baseline and 1-month follow-up $(\mathrm{p}<.001)$ and EOI and 1-month follow-up $(\mathrm{p}<.05)$.

Statistical significance was also found across time on BIAQ scores, $\mathrm{F}_{(2,23)}=26.64, \mathrm{p}<.001$, $\eta^{2}=.70$. Pairwise comparisons indicated a significant decrease between the BIAQ scores between baseline and EOI $(\mathrm{p}<.001)$ and baseline and 1-month follow-up $(\mathrm{p}<.01)$. However, there was a significant increase between EOI and 1-month follow-up $(\mathrm{p}<.01)$.

There was no significant time effect for Quality of life, $F(2,28)=.75, \mathrm{p}>.5, \mathrm{n}^{2}=.03$.

Odds ratios for meeting criteria for at-risk at EOI

At EOI, 57.10\% ( $n=14)$ of participants, and at -month follow-up, $72 \%(n=18)$ of participants, no longer met criteria for being at high-risk for ED development (i.e., WCS score<52; Killen et al., 1994). Chi-square analysis was statistically significant $(\mathrm{p}<.001)$ with a value of 16.75 , and the risk analysis results showed the odds of falling into the atrisk group (WCS>52) at EOI were 14.5 times higher for participants in the control vs. the AcceptME group (CI: 3.15, 66.67).

\subsection{Figures and Tables}

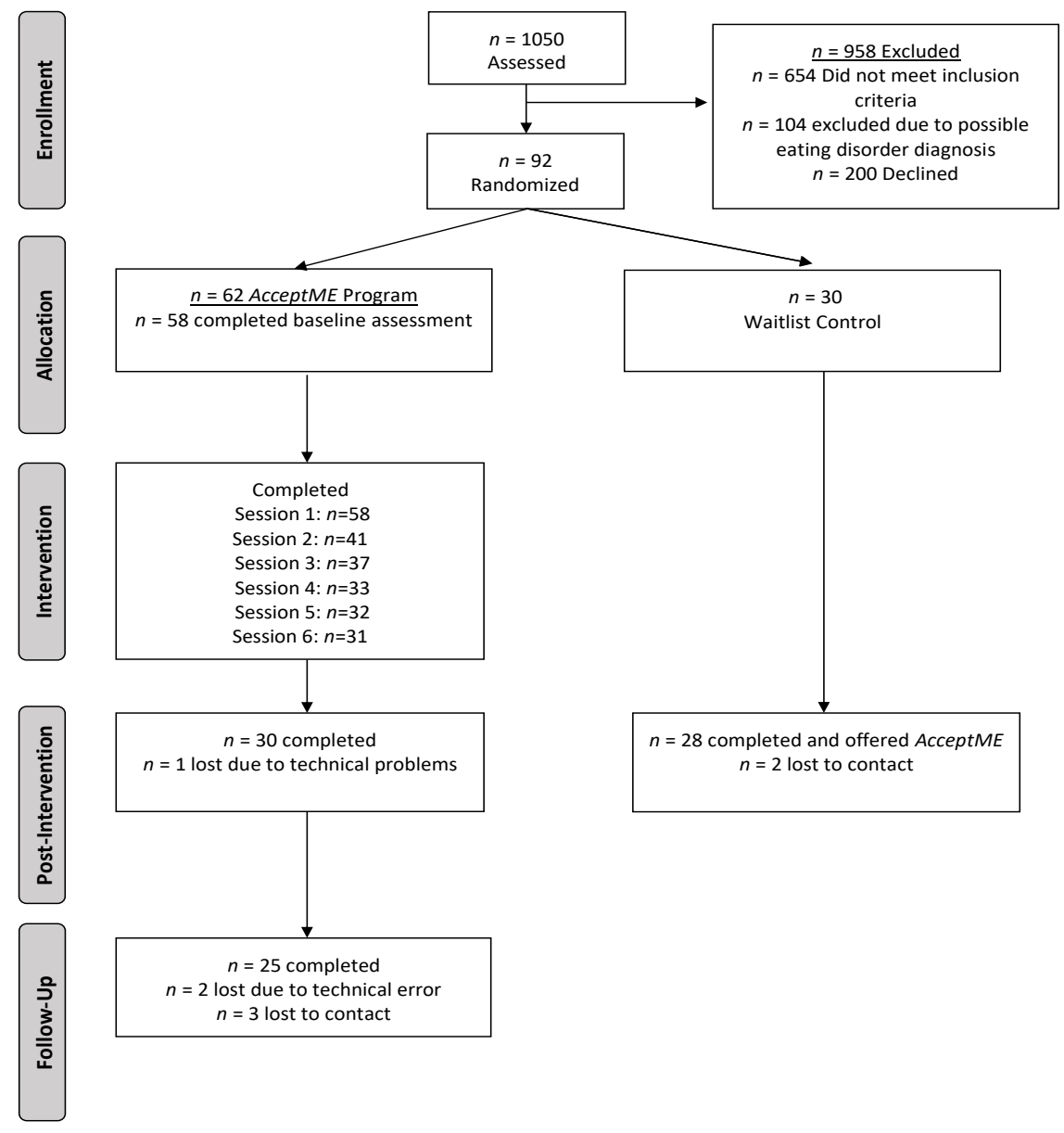

Figure 1. CONSORT flow diagram 


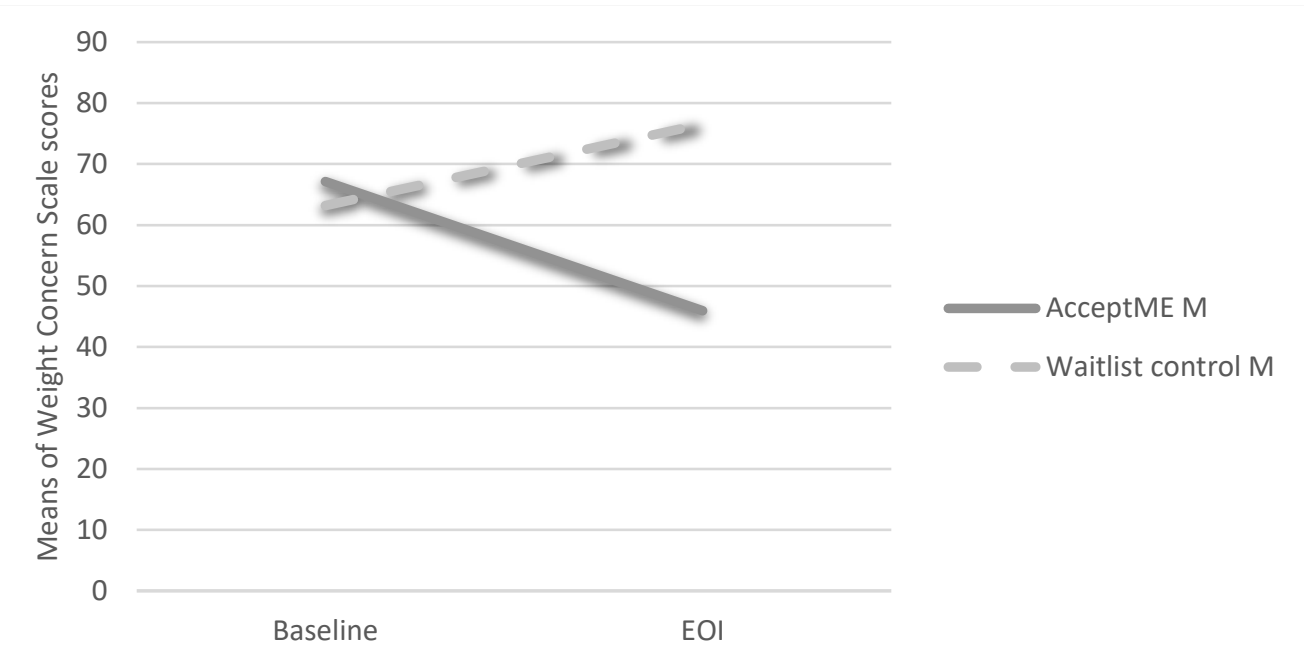

Figure 2a. Group by Time Interaction of Weight Concern Scale scores

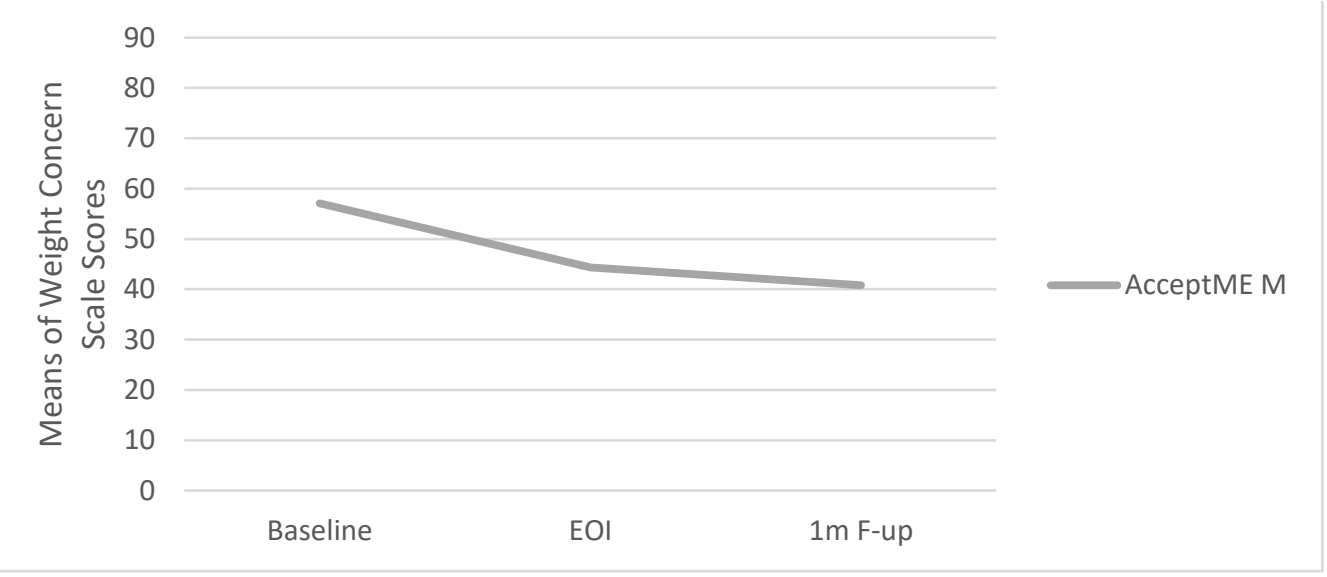

Figure 2b. Weight Concern Scale scores across time only for AcceptME group 


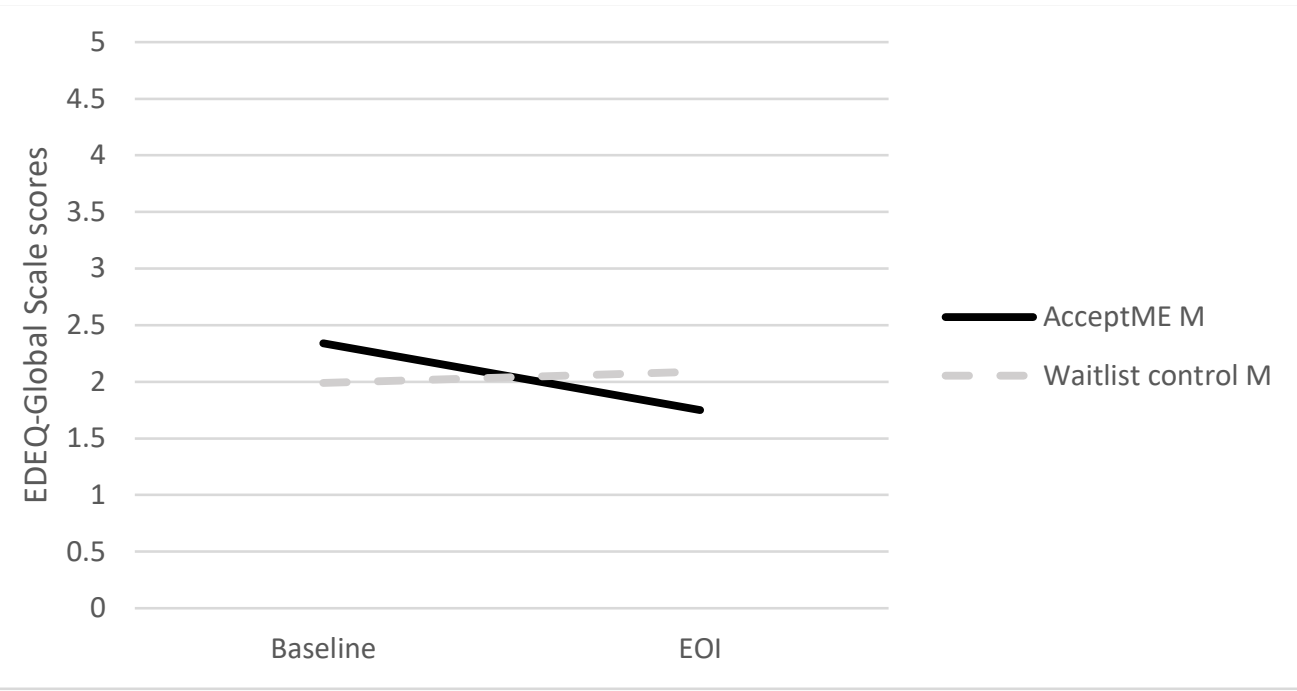

Figure 3a. Group by Time Interaction of EDEQ-Global score

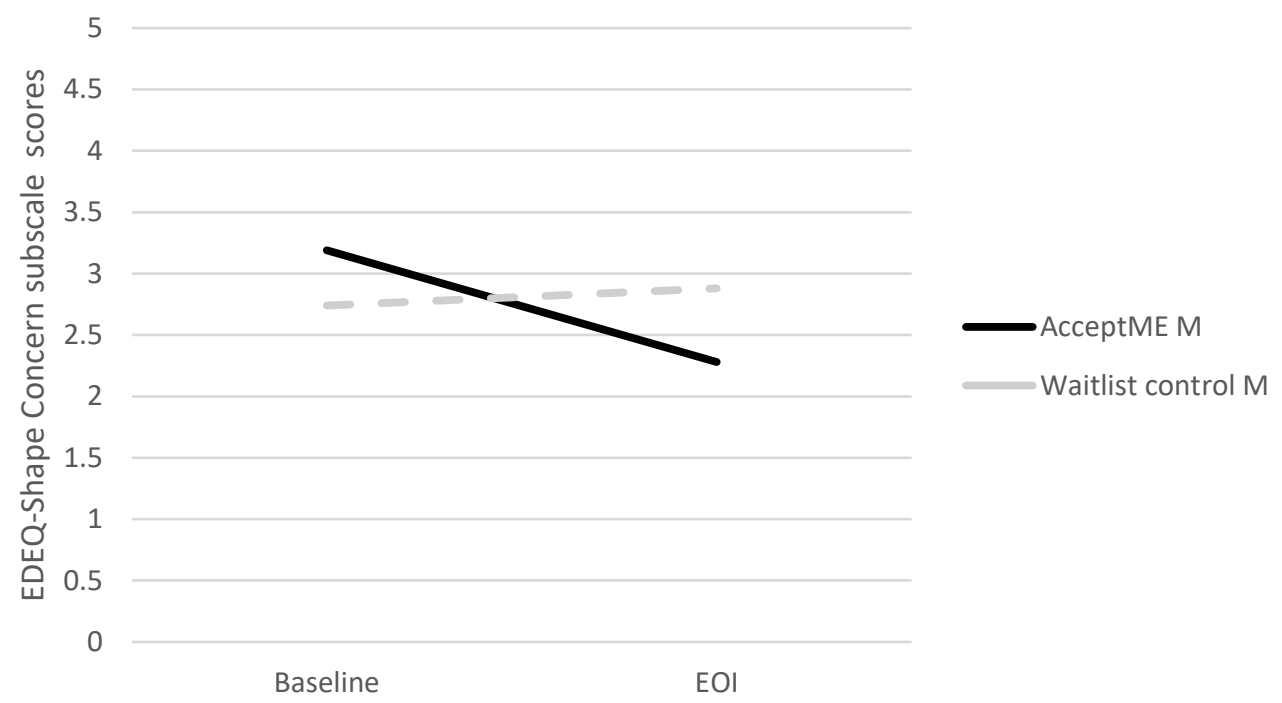

Figure 3b. Group by Time Interaction of EDEQ- Shape concern subscale scores

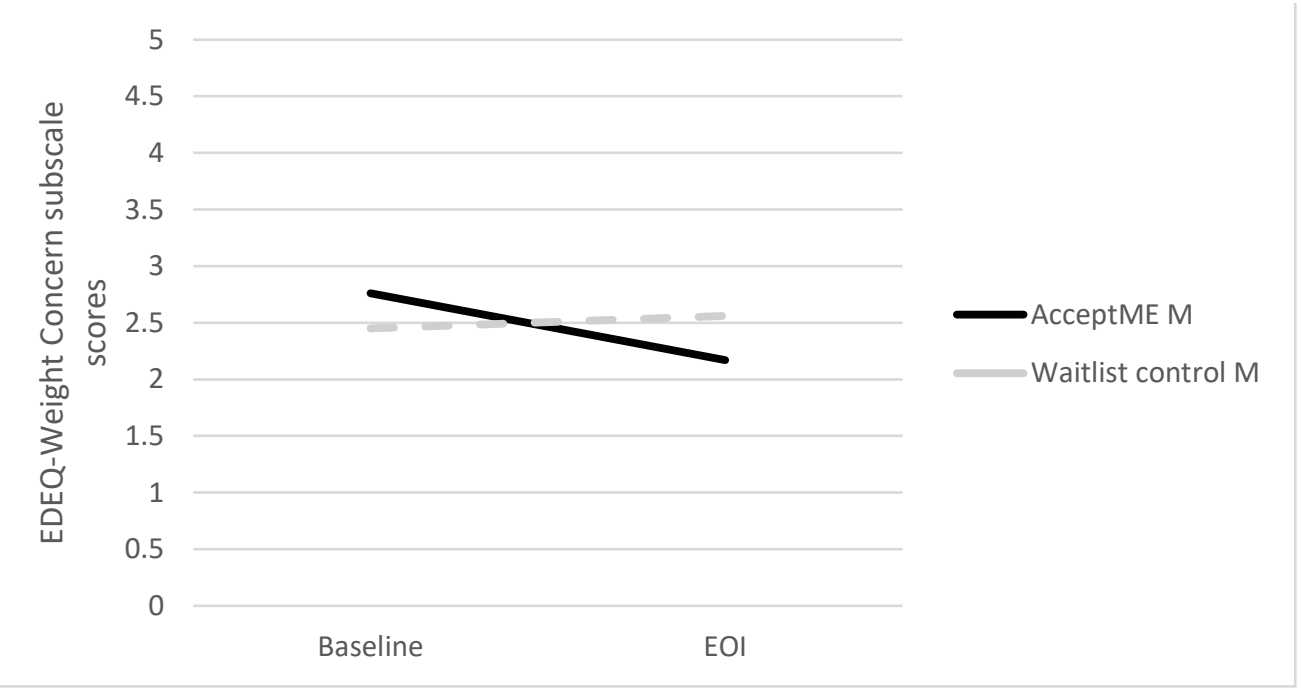

Figure 3c. Group by Time Interaction of EDEQ- Weight concern subscale scores 


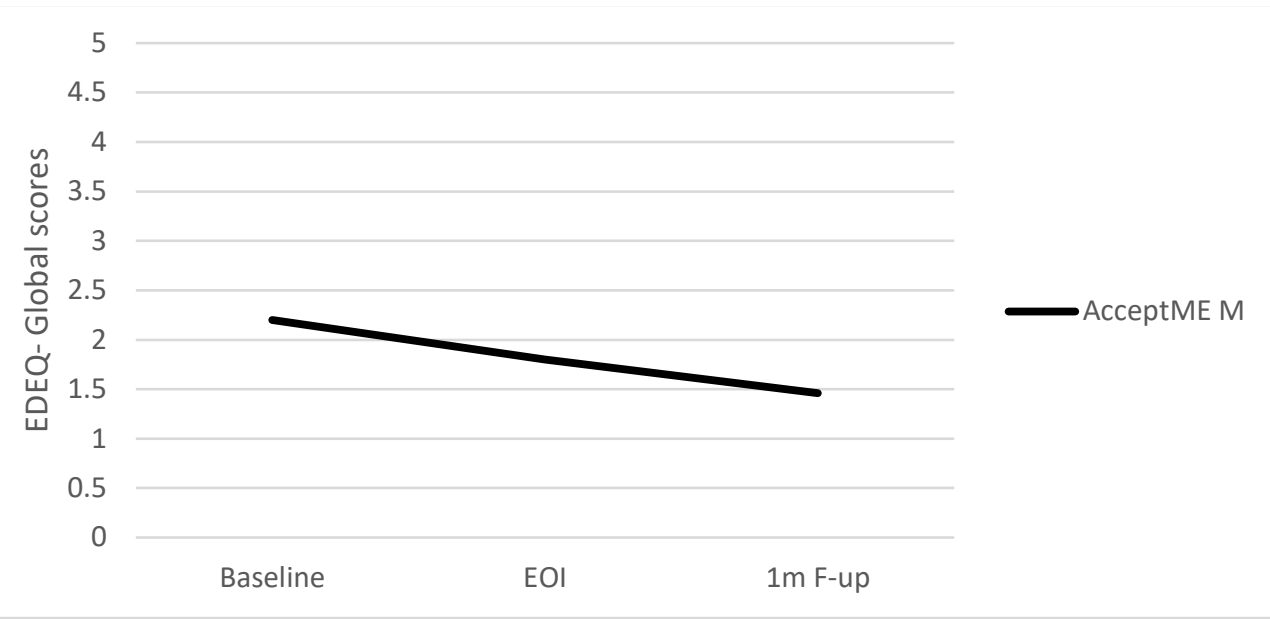

Figure 3d. EDEQ-Global scores for AcceptME across time

Table 1. Means and standard deviations of outcome at baseline and end of intervention by group (AcceptME vs. waitlist control)

\begin{tabular}{|c|c|c|c|c|c|}
\hline & \multicolumn{2}{|c|}{ ACT Group } & \multicolumn{2}{|c|}{ Control Group } & Interaction effects \\
\hline & Baseline & EOI & Baseline & EOI & \\
\hline & $\mathrm{M}(\mathrm{SD})$ & $\mathrm{M}(\mathrm{SD})$ & $\mathrm{M}(\mathrm{SD})$ & $\mathrm{M}(\mathrm{SD})$ & \\
\hline & $N=59$ & $\mathrm{~N}=29$ & $\mathrm{~N}=29$ & $\mathrm{~N}=28$ & \\
\hline \multirow[t]{2}{*}{ WCS } & 67.14 & 45.95 & 63.19 & 76.54 & $F_{(1,54)}=56.67$ \\
\hline & (11.93) & $(25.90)$ & $(8.58)$ & $(14.85)$ & $\eta^{2}=.52$ \\
\hline$\%$ score $<52$ high-risk cut-off & $0 \%$ & $57.10 \%$ & $0 \%$ & $7.10 \%$ & \\
\hline \multicolumn{6}{|l|}{ EDE-Q } \\
\hline Restraint Subscale & $1.82(1.34)$ & $1.37(1.06)$ & $1.64(1.19)$ & $1.70(.95)$ & NS \\
\hline Eating Concern Subscale & $1.59(1.06)$ & $1.22(1.02)$ & $1.37(.92)$ & $1.27(.84)$ & NS \\
\hline Shape Concern Subscale & $3.18(1.21)$ & $2.28(1.33)$ & $2.74(1.07)$ & $2.88(1.05)$ & $F_{(1,54)}=8.49, p<.01, \eta^{2}=.14$ \\
\hline Weight Concern Subscale & $2.76(1.07)$ & $2.17(1.01)$ & $2.45(1.00)$ & $2.56(1.01)$ & $F_{(1,54)}=3.81, p=.05, \eta^{2}=.07$ \\
\hline Global & $2.34(.93)$ & $1.75(.99)$ & $1.99(.91)$ & $2.09(.66)$ & NS \\
\hline \multirow[t]{2}{*}{ YQOL-SF } & 66.06 & 62.71 & 68.06 & 68.21 & NS \\
\hline & $(10.05)$ & $(22.57)$ & (11.86) & (10.10) & \\
\hline \multirow[t]{2}{*}{ BSQ-8C } & 29.93 & 23.34 & 26.54 & 26.27 & NS \\
\hline & $(8.35)$ & (11.27) & (10.01) & (11.35) & \\
\hline
\end{tabular}




$\begin{array}{llllll}\text { BI-AAQ } & 48.45 & 56.76 & 48.41 & 50.85 & \text { NS } \\ & (16.90) & (15.54) & (13.32) & (15.10) & \\ \text { BIAQ } & 35.44 & 19.75(8.58) & 37.54 & 23.86 & \text { NS } \\ & (11.62) & & (18.45) & (11.05) & \end{array}$

Note: EOI= End of Intervention time point of assessment; WCS=Weight Concerns Scale; EDE-Q=Eating Disorder Examination Questionnaire; YQOL-SF=Youth Quality of Life-Short Form; BSQ8C=Body Shape Questionnaire-8C; BI-AAQ=Body Image-Acceptance and Action Questionnaire; $B I A Q=B o d y$ Image Avoidance Questionnaire; NS=Non statistically significance difference with $\mathrm{p}>.05$.

Table 2. Means and standard deviations of outcomes at baseline, EOI, and one month follow-up for the AcceptME group.

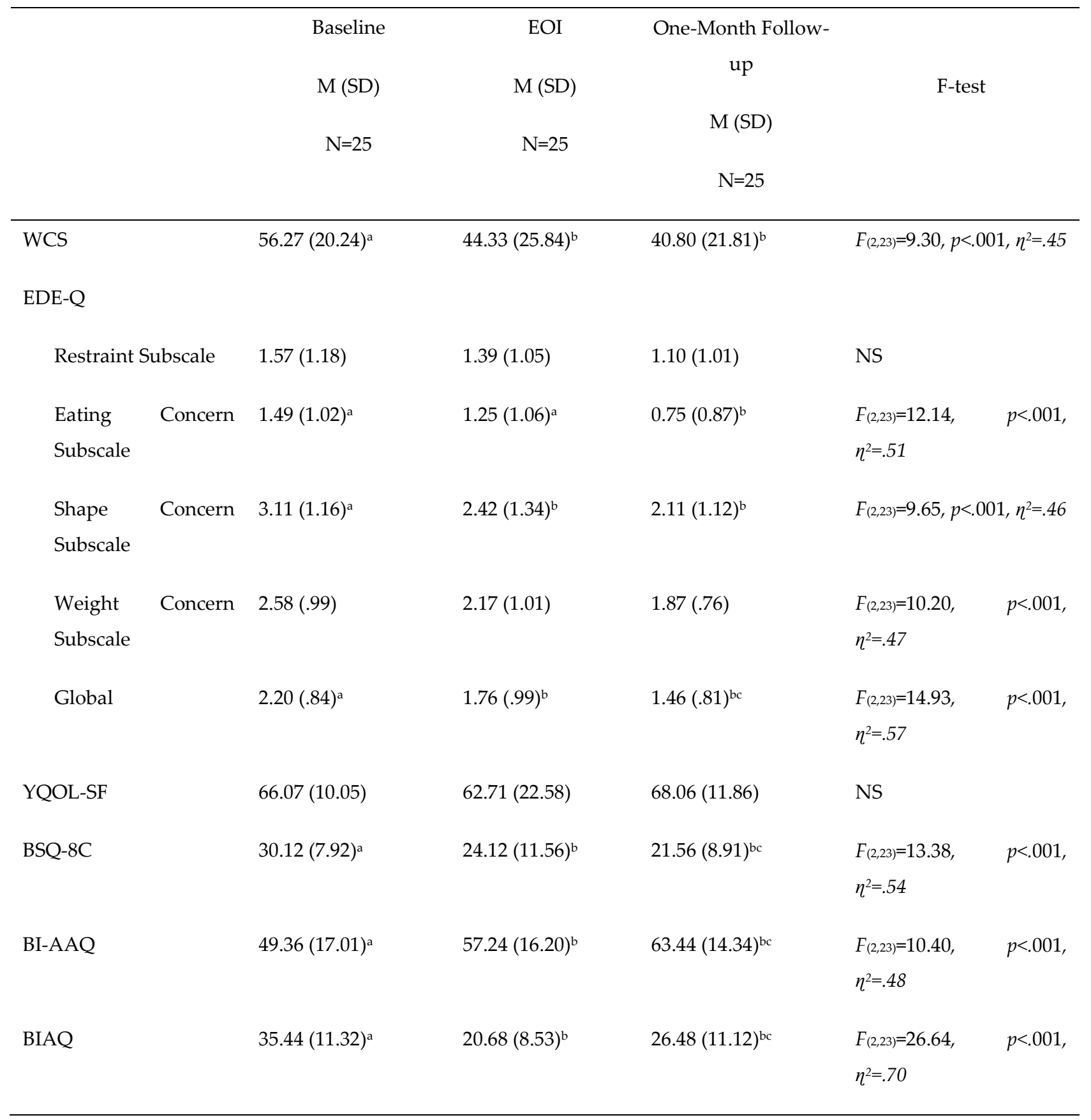


Note $1{ }^{a}$ significantly different from ${ }^{b}$ and ${ }^{b}$ significantly different from ${ }^{c}$.

Note 2: EOI= End of Intervention; WCS=Weight Concerns Scale; EDE-Q=Eating Disorder Examination Questionnaire; YQOL-SF=Youth Quality of Life Instrument-Short Form; BSQ-8C=Body Shape Questionnaire-8C; BI-AAQ=Body Image-Acceptance and Action Questionnaire; and BIAQ=Body Image Avoidance Questionnaire.

\section{Discussion}

This is the first study to develop and test an innovative digital, gamified ACT-based ED early-intervention program for youth showing signs and symptoms of an ED and being at high-risk for the development of full syndrome. Consistent with our hypothesis, young women who participated in the AcceptME program had significantly lower weight and shape concerns at the end of the program in comparison to participants in the waitlist control group with large effect sizes. This is consistent with at least one other acceptance and mindfulness-based digital program for EDs. Atkinson and colleagues [43], for example, assessed a mindfulness-based intervention to reduce the risk for ED development and their results showed significant reductions in WCS with large effect size. These effect sizes exceed the medium effect sizes reported by other programs such as the digital cognitive dissonance program $[44,45]$. Present study findings are promising and support the continued development of ACT-based early-interventions to reduce ED symptoms in young females at-risk for EDs.

Most young women who completed the AcceptME intervention scored below the cut-off score for being in the at-risk category for developing an ED in the next four years at endof-intervention (57.10\%; WCS score $<52$ ) [29]. Interestingly, $72 \%$ of intervention completers scored below this cut-off at the 1-month follow-up. The average mean score on the WCS $(M=45.95)$ was overall below the cut-off with evidenced additional reductions in WCS scores $(M=40.80)$ at the 1-month follow-up. In contrast, young women in the waitlist control group had a statistically significant increase in weight and shape concerns $(M=67.14)$ with only $7.1 \%$ scoring below the at-risk cut-off at EOI presenting them with 14.5 times higher odds of being at-risk of developing an ED compared to the AcceptME group.

The AcceptME group also had significantly lower scores on the global EDE-Q, as well as the weight and shape concern subscales. These subscales assess some of the cognitive features of ED symptoms, suggesting that participants became less preoccupied with weight and shape after their participation in the program. Improvements in ED symptoms appeared to be maintained at the 1-month follow-up period with large effect sizes. However, these findings should be interpreted with caution given the lack of comparison group for the 1-month follow-up. Taken together, the results of the current study support that ACT-based skills learned in this novel digital, gamified ED early-intervention program, can reduce ED symptomatology and parameters associated with the risk for ED development.

For some outcomes, there were no interaction effects but significant time effects for the AcceptME group, with continued improvement over time to the 1-month follow-up. Body image flexibility, notably showed continued improvement over time. This may reflect additional consolidation of acceptance-based concepts, consistent with other studies that have shown delayed but potentially more persistent effects in ACT interventions. However, long-term follow-up with a control condition is needed.

No significant effects were noted for quality of life. The scores reported on the YQOL-SF were close to those reported for general population of adolescents who do not present significant psychopathology (e.g., [46,47]) thus it may be that participants in the present study did not experience significant quality of life impacts and thus there was not much 
room for improvement. The impacts on quality of life of youth presenting at high-risk for developing an ED warrants further research examination.

This is the first study to assess this kind of a digital, gamified ACT early-intervention program for individuals at high-risk for developing an ED, utilizing also a non-threatening vicarious learning approach. The large effect sizes for key outcomes is of note. Unlike some prevention and early-intervention programs, this program, based on ACT principles, takes into consideration and attempts to alter the function that ED symptoms serve for the individual. This includes the negative functions, such as removal of unwanted internal experiences, as well as the positive experiences of mastery or pride with successful eating and weight control $[48,49]$. The program helps the individual recognize these different functions how efforts to control or manipulate their internal experiences may work in the short-term but be ineffective in the long-run or for the individuals' personal values. Through the storyline and exercises, individuals learn new ways of responding to unwanted thoughts and feelings, and via engagement with values, contact alternative reinforcers.

An additional strength of the intervention is the digital format, which allowed individuals to participate at their own time and place. Also, since the program is web-based delivered, it could be easily implemented and disseminated to potential users in the future. Once refined, the digital prevention program can be considered by high schools, colleges or universities to reduce ED symptom progression. Further research and replication, and implementation research in other contexts is important and warranted.

Although these results are promising, future investigation is needed to clarify the specific treatment components (e.g., increased motivation, values clarification, psychological flexibility, cognitive defusion) responsible for therapeutic effects with explicit exploration of the proposed mechanisms of change.

\subsection{Limitations of the study}

A key limitation of the study was that group comparisons were only able to be conducted at end-of-intervention, as participants in the control group were provided access to the treatment at that point. It would be beneficial to evaluate the AcceptME program in a study with a longer-term follow-up. Longer-term follow-up would have been especially useful as the results suggested additional improvements in psychological flexibility and ED symptoms to occur between the post and the one-month follow up time points. The study was also limited by the use of waitlist control group instead of another active intervention for comparison. The use of another treatment as a control was difficult in the present study, as no similar already established digital intervention in Greek language was found. Finally, the sample was relatively homogenous with regards to ethnicity, suggesting that care should be taken in generalizing the results to more diverse populations. It would be beneficial to have the program translated in other languages so that experts on ACT and ED can review and provide fidelity evidence for the program and to further assess the program in other ethnic, racial and cultural groups. Another limitation involves the number of individuals who were recognized to be athigh risk but did not consent to take part in the intervention trial. Although it was expected that this is a difficult population to engage in any sort of intervention program, future attempts need to be made to motivate individuals deemed to be at-risk to engage in such intervention programs. Also, non-completion once individuals enter an intervention is another important issue that needs to be considered and a limitation of the study. Though the percent of non-completion is similar to previous similar studies (Karekla et al., 2020; Saekow et al., 2015; Taylor et al., 2021), and ITT analyses did corroborate the findings of the trial, caution in interpretation is needed. Despite these limitations, the 
results of the study suggest that this is a promising new approach even if further research is necessary.

\section{Conclusions}

In conclusion, the current study raises the possibility that an ACT-based digital gamified early-intervention program may be effective for young women with early signs and symptoms of an ED, and at-risk for ED progression. More research is needed to replicate and extend these early findings.

Author Contributions: Conceptualization, M.K. and P.N.; Methodology, M.K., P.N., and R.M.; Software, M.K. and P.N.; Formal Analysis, M.K. and P.N.; Investigation, M.K. and P.N.; Resources, M.K.; Data Curation, M.K. and P.N.; Writing - Original Draft Preparation, M.K., and P.N.; Writing - Review \& Editing, M.K., P.N., and R.M.; Visualization, M.K.; Supervision,M.K.; Project Administration, M.K. and P.N.; Funding Acquisition, M.K.

Funding: Development of the digital program was supported by University of Cyprus funds (Startup grant and Research allowance) provided to Dr. Maria Karekla.

Institutional Review Board Statement: All procedures were in accordance with national and international ethical standards. The study was approved from the Cyprus National Bioethics Committee (ЕЕВК/ЕП/2013/05) and the Cyprus Ministry of Education and Culture.

Informed Consent Statement: Informed consent was obtained from all participants involved in the study. In the case of individuals under 18, consent was also secured from their parents or legal guardians.

Data Availability Statement: Data are available from the corresponding author upon request.

Acknowledgments: Part of this paper was submitted for fulfilling the doctoral requirements of the second author. The authors would like to thank the Cyprus Ministry of Education Coalition for Health Promotion in Schools for their help in sample recruitment and Pinelopi Konstantinou for her help with referencing.

Conflicts of Interest: All authors declare that they have no conflicts of interest.

\section{References}

1. Stice, E.; Becker, C.B.; Yokum, S. Eating disorder prevention: Current evidence-base and future directions. Int. J. Eat. Disord. 2013, 46, 478-485, doi:10.1002/eat.22105.

2. Vanderlinden, J. Many roads lead to Rome: Why does cognitive behavioural therapy remain unsuccessful for many eating disorder patients? Eur. Eat. Disord. Rev. 2008, 16, 329-333, doi:10.1002/erv.889.

3. Fairburn, C.G.; Cooper, Z.; Doll, H.A.; O'Connor, M.E.; Palmer, R.L.; Dalle Grave, R. Enhanced cognitive behaviour therapy for adults with anorexia nervosa: A UK-Italy study. Behav. Res. Ther. 2013, 51, R2-R8, doi:10.1016/j.brat.2012.09.010.

4. Manlick, C.F.; Cochran, S. V.; Koon, J. Acceptance and commitment therapy for eating disorders: Rationale and literature review. J. Contemp. Psychother. 2013, 43, 115-122, doi:10.1007/s10879-012-9223-7.

5. Merwin, R.M.; Timko, C.A.; Moskovich, A.A.; Ingle, K.K.; Bulik, C.M.; Zucker, N.L. Psychological inflexibility and Symptom Expression in Anorexia Nervosa. Eat. Disord. 2011, 19, 62-82, doi:10.1080/10640266.2011.533606.

6. Koushiou, M.; Nicolaou, K.; Karekla, M. Inducing negative affect using film clips with general and eating disorder-related content. Eat. Weight Disord. 2018, doi:10.1007/s40519-018-0485-2.

7. Hayes, S.C.; Strosahl, K.D.; Wilson, K.G. Acceptance and commitment therapy: An experimental approach to behavior change; 1999; ISBN 1572309555.

8. Hayes, S.C.; Strosahl, K.D.; Wilson, K.G. Acceptance and commitment therapy: The process and practice of mindful change; New York, NY, US, 2011; ISBN 978-1-60918-962-4 (Hardcover); 978-1-60918-964-8 (PDF).

9. Hayes, S.C.; Luoma, J.B.; Bond, F.W.; Masuda, A.; Lillis, J. Acceptance and Commitment Therapy: Model, processes and outcomes. Behav. Res. Ther. 2006, 44, 1-25, doi:10.1016/j.brat.2005.06.006.

10. Linardon, J.; Fairburn, C.G.; Fitzsimmons-Craft, E.E.; Wilfley, D.E.; Brennan, L. The empirical status of the third-wave behaviour therapies for the treatment of eating disorders: A systematic review. Clin. Psychol. Rev. 2017, 58, 125-140, doi:10.1016/j.cpr.2017.10.005.

11. Linardon, J.; Gleeson, J.; Yap, K.; Murphy, K.; Brennan, L. Meta-analysis of the effects of third-wave behavioural interventions on disordered eating and body image concerns: implications for eating disorder prevention. Cogn. Behav. Ther. 2019, 48, 15-38, doi:10.1080/16506073.2018.1517389. 
12. Linardon, J.; Shatte, A.; Messer, M.; Firth, J.; Fuller-Tyszkiewicz, M. E-mental health interventions for the treatment and prevention of eating disorders: An updated systematic review and meta-analysis. J. Consult. Clin. Psychol. 2020, 88, 994-1007, doi:10.1037/ccp0000575.

13. Juarascio, A.; Shaw, J.; Forman, E.; Timko, C.A.; Herbert, J.; Butryn, M.; Bunnell, D.; Matteucci, A.; Lowe, M. Acceptance and Commitment Therapy as a Novel Treatment for Eating Disorders: An Initial Test of Efficacy and Mediation. Behav. Modif. 2013, 37, 459-489, doi:10.1177/0145445513478633.

14. Pearson, A.N.; Follette, V.M.; Hayes, S.C. A Pilot Study of Acceptance and Commitment Therapy as a Workshop Intervention for Body Dissatisfaction and Disordered Eating Attitudes. Cogn. Behav. Pract. 2012, 19, 181-197, doi:10.1016/j.cbpra.2011.03.001.

15. Juarascio, A.S.; Forman, E.M.; Herbert, J.D. Acceptance and commitment therapy versus cognitive therapy for the treatment of comorbid eating pathology. Behav. Modif. 2010, 34, 175-190, doi:10.1177/0145445510363472.

16. Timko, C.A.; Zucker, N.L.; Herbert, J.D.; Rodriguez, D.; Merwin, R.M. An open trial of Acceptance-based Separated Family Treatment (ASFT) for adolescents with anorexia nervosa. Behav. Res. Ther. 2015, 69, 63-74, doi:10.1016/j.brat.2015.03.011.

17. Pinto-Gouveia, J.; Carvalho, S.A.; Palmeira, L.; Castilho, P.; Duarte, C.; Ferreira, C.; Duarte, J.; Cunha, M.; Matos, M.; Costa, J. Incorporating psychoeducation, mindfulness and self-compassion in a new programme for binge eating (BEfree): Exploring processes of change. J. Health Psychol. 2019, 24, 466-479, doi:10.1177/1359105316676628.

18. Fogelkvist, M.; Gustafsson, S.A.; Kjellin, L.; Parling, T. Acceptance and commitment therapy to reduce eating disorder symptoms and body image problems in patients with residual eating disorder symptoms: A randomized controlled trial. Body Image 2020, 32, 155-166, doi:10.1016/j.bodyim.2020.01.002.

19. Parling, T.; Cernvall, M.; Ramklint, M.; Holmgren, S.; Ghaderi, A. A randomised trial of Acceptance and Commitment Therapy for Anorexia Nervosa after daycare treatment, including five-year follow-up. BMC Psychiatry 2016, 16, 1-13, doi:10.1186/s12888016-0975-6.

20. Pellizzer, M.L.; Waller, G.; Wade, T.D. Body image flexibility: A predictor and moderator of outcome in transdiagnostic outpatient eating disorder treatment. Int. J. Eat. Disord. 2018, 51, 368-372, doi:10.1002/eat.22842.

21. Rogers, C.B.; Webb, J.B.; Jafari, N. A systematic review of the roles of body image flexibility as correlate, moderator, mediator, and in intervention science (2011-2018). Body Image 2018, 27, 43-60, doi:10.1016/j.bodyim.2018.08.003.

22. Stice, E.; Shaw, H.; Marti, C.N. A meta-analytic review of eating disorder prevention programs: Encouraging findings. Annu. Rev. Clin. Psychol. 2007, 3, 207-231, doi:10.1146/annurev.clinpsy.3.022806.091447.

23. Hoek, H.W.; Van Hoeken, D. Review of the Prevalence and Incidence of Eating Disorders. Int. J. Eat. Disord. 2003, 34, 383-396, doi:10.1002/eat.10222.

24. Dölemeyer, R.; Tietjen, A.; Kersting, A.; Wagner, B. Internet-based interventions for eating disorders in adults: A systematic review. BMC Psychiatry 2013, 13, doi:10.1186/1471-244X-13-207.

25. Newton, M.S.; Ciliska, D. Internet-based innovations for the prevention of eating disorders: A systematic review. Eat. Disord. 2006, 14, 365-384, doi:10.1080/10640260600952522.

26. Kahn, J.; Ducharme, P.; Rotenberg, A.; Gonzalez-Heydrich, J. "rAGE-Control": A Game to Build Emotional Strength. Games Health J. 2013, 2, 53-57, doi:10.1089/g4h.2013.0007.

27. Deterding, S.; Dixon, D.; Khaled, R.; Nacke, L. From game design elements to gamefulness: Defining "gamification." Proc. 15th Int. Acad. MindTrek Conf. Envisioning Futur. Media Environ. MindTrek 2011 2011, 9-15, doi:10.1145/2181037.2181040.

28. Karekla, M.; Kasinopoulos, O.; Neto, D.D.; Ebert, D.D.; Van Daele, T.; Nordgreen, T.; Höfer, S.; Oeverland, S.; Jensen, K.L. Best Practices and Recommendations for Digital Interventions to Improve Engagement and Adherence in Chronic Illness Sufferers. Eur. Psychol. 2019, 24, 49-67, doi:10.1027/1016-9040/a000349.

29. Killen, J.D.; Hayward, C.H.; Haydel, F.; Wilson, D.; Hammer, L.; Kraemer, H.; Blair-Greiner, A.; Strachowski, D. Weight concerns influence the development of eating disorders. J. Consult. Clin. Psychol. 1996, 64, 936-940.

30. Stice, E.; Telch, C.F.; Rizvi, S.L. Development and validation of the eating disorder diagnostic scale: A brief self-report measure of anorexia, bulimia, and binge-eating disorder. Psychol. Assess. 2000, 12, 123-131, doi:10.1037/1040-3590.12.2.123.

31. Fairburn, C.G.; Norman, P.A.; Welch, S.L.; O'Connor, M.E.; Doll, H.A.; Peveler, R.C. A Prospective Study of Outcome in Bulimia Nervosa and the Long-term Effects of Three Psychological Treatments. Arch. Gen. Psychiatry 1995, 52, 304-312, doi:10.1001/archpsyc.1995.03950160054010.

32. Rizvi, S.L.; Peterson, C.B.; Crow, S.J.; Agras, W.S. Test-retest reliability of the Eating Disorder Examination. Int. J. Eat. Disord. 2000, 28, 311-316, doi:10.1002/1098-108X(200011)28:3<311::AID-EAT8>3.0.CO;2-K.

33. Rosen, J.C.; Vara, L.; Wendt, S.; Leitenberg, H. Validity studies of the eating disorder examination. Int. J. Eat. Disord. 1990, 9, 519-528, doi:10.1002/1098-108X(199009)9:5<519::AID-EAT2260090507>3.0.CO;2-K.

34. Fairburn, C.G.; Beglin, S.J. Assessment of eating disorders: Interview or self-report questionnaire? Int. J. Eat. Disord. 1994, 16, 363-370, doi:10.1002/1098-108X(199412)16:4<363::AID-EAT2260160405>3.0.CO;2-\#.

35. Juarascio, A.; Shaw, J.; Forman, E.M.; Timko, C.A.; Herbert, J.D.; Butryn, M.L.; Lowe, M. Acceptance and Commitment Therapy for eating disorders: Clinical applications of a group treatment. J. Context. Behav. Sci. 2013, 2, 85-94, doi:10.1016/j.jcbs.2013.08.001.

36. Patrick, D.L.; Edwards, T.C.; Topolski, T.D. Adolescent quality of life, part II: Initial validation of a new instrument. J. Adolesc. 2002, 25, 287-300, doi:10.1006/jado.2002.0471.

37. Cooper, P.J.; Taylor, M.J.; Cooper, Z.; Fairbum, C.G. The development and validation of the body shape questionnaire. Int. J. Eat. Disord. 1987, 6, 485-494, doi:10.1002/1098-108X(198707)6:4<485::AID-EAT2260060405>3.0.CO;2-O.

38. Pook, M.; Tuschen-Caffier, B.; Brähler, E. Evaluation and comparison of different versions of the Body Shape Questionnaire. Psychiatry Res. 2008, 158, 67-73, doi:10.1016/j.psychres.2006.08.002. 
39. Welch, E.; Lagerström, M.; Ghaderi, A. Body Shape Questionnaire: Psychometric properties of the short version (BSQ-8C) and norms from the general Swedish population. Body Image 2012, 9, 547-550, doi:10.1016/j.bodyim.2012.04.009.

40. Sandoz, E.K.; Wilson, K.G.; Merwin, R.M.; Kate Kellum, K. Assessment of body image flexibility: The Body Image-Acceptance and Action Questionnaire. J. Context. Behav. Sci. 2013, 2, 39-48, doi:10.1016/j.jcbs.2013.03.002.

41. Karekla, M.; Mavraki, E.Z.; Nikolaou, P.; Koushiou, M. Validation of the greek version of the body image-acceptance and action questionnaire. Eur. J. Couns. Psychol. 2020, 8, 93-107, doi:10.5964/ejcop.v8i1.173.

42. Rosen, J.C.; Srebnik, D.; Saltzberg, E.; Wendt, S. Development of a body image avoidance questionnaire. - consequences (body image avoiding behaviours). Psychol. Assess. 1991, 3, 32-37.

43. Atkinson, M.J.; Wade, T.D. Does mindfulness have potential in eating disorders prevention? A preliminary controlled trial with young adult women. Early Interv. Psychiatry 2016, 10, 234-245, doi:10.1111/eip.12160.

44. Jacobi, C.; Völker, U.; Trockel, M.T.; Taylor, C.B. Effects of an Internet-based intervention for subthreshold eating disorders: A randomized controlled trial. Behav. Res. Ther. 2012, 50, 93-99, doi:10.1016/j.brat.2011.09.013.

45. Kass, A.E.; Trockel, M.; Safer, D.L.; Sinton, M.M.; Cunning, D.; Rizk, M.T.; Genkin, B.H.; Weisman, H.L.; Bailey, J.O.; Jacobi, C.; et al. Internet-based preventive intervention for reducing eating disorder risk: A randomized controlled trial comparing guided with unguided self-help. Behav. Res. Ther. 2014, 63, 90-98, doi:10.1016/j.brat.2014.09.010.

46. Cotter, E.W.; Hornack, S.E.; Fotang, J.P.; Pettit, E.; Mirza, N.M. A pilot open-label feasibility trial examining an adjunctive mindfulness intervention for adolescents with obesity. Pilot Feasibility Stud. 2020, 6, 1-11, doi:10.1186/s40814-020-00621-1.

47. Hoang, M.T.; Do, H.N.; Dang, T.Q.; Do, H.T.; Nguyen, T.T.; Nguyen, L.H.; Nguyen, C.T.; Doan, L.P.; Vu, G.T.; Van Ngo, T.; et al. Cross-cultural adaptation and measurement properties of Youth Quality of Life Instrument- Short Form (YQOL-SF) in a developing South- East Asian country. PLoS One 2021, 16, 1-15, doi:10.1371/journal.pone.0253075.

48. Merwin, R.M.; Wilson, K. Understanding and Treating Eating Disorders: An ACT perspective. In Acceptance and commitment therapy: Contemporary theory, research, and practice; Australian Academic Press: Sydney, 2009; pp. 87-117 ISBN 9781921513145.

49. Merwin, R.M.; Zucker, N.L.; Wilson, K.G. ACT for anorexia nervosa: A guide for clinicians.; The Guilford Press: New York, NY, US, 2019; ISBN 9781462540341 (Paperback); 9781462540358 (Hardcover). 